\title{
Clinical Response and Quality of Life in Patients with Severe Atopic Dermatitis Treated with Dupilumab: A Single-Center Real-Life Experience
}

\author{
Silvia Ferrucci ${ }^{1}$, Giovanni Casazza ${ }^{2}$, , Luisa Angileri ${ }^{1,3}$, Simona Tavecchio ${ }^{1,3}$, \\ Francesca Germiniasi ${ }^{1,3}$, Emilio Berti ${ }^{1,3}$, Angelo Valerio Marzano ${ }^{1,3}$ (D) and \\ Giovanni Genovese ${ }^{1,3, *(1)}$ \\ 1 UOC Dermatologia, Fondazione IRCCS Ca' Granda Ospedale Maggiore Policlinico, 20122 Milan, Italy; \\ silvia.ferrucci@policlinico.mi.it (S.F.); luisa.angileri@unimi.it (L.A.); simona.tavecchio@gmail.com (S.T.); \\ francesca.germiniasi@unimi.it (F.G.); emilio.berti@unimi.it (E.B.); angelo.marzano@unimi.it (A.V.M.) \\ 2 Dipartimento di Scienze Biomediche e Cliniche "L. Sacco", Università degli Studi di Milano, 20157 Milan, \\ Italy; giovanni.casazza@unimi.it \\ 3 Dipartimento di Fisiopatologia Medico-Chirurgica e dei Trapianti, Università degli Studi di Milano, \\ 20122 Milan, Italy \\ * Correspondence: giov.genov@gmail.com; Tel.: +39-025-503-4707; Fax: +39-025-503-5236
}

Received: 12 February 2020; Accepted: 12 March 2020; Published: 13 March 2020

\begin{abstract}
Dupilumab is an anti-interleukin-4 receptor monoclonal antibody that was recently approved for the treatment of atopic dermatitis (AD). In this single-center retrospective study, clinical baseline data of 117 severe AD patients treated with dupilumab were collected. At baseline and at weeks 4 and 16, disease severity was assessed through the Eczema Area and Severity Index (EASI) and quality of life through the Dermatology Life Quality Index (DLQI) questionnaire, Patient-Oriented Eczema Measure (POEM), Hospital Anxiety and Depression Scale (HADS), Peak Pruritus Numerical Rating Scale (NRS-itch), and VAS-sleep. Response to dupilumab was defined as animprovement of $\geq 75 \%$ in EASI from baseline (EASI75). At multivariate analysis, AD onset before 18 years [OR, 2.9; $95 \%$ CI, 1.2-7.2; $p=0.0207$ ] and absence of hypereosinophilia [OR, 2.24; 95\% CI, 1.03-4.86; $p=0.0412]$ were identified as significant predictive parameters for response to dupilumab in terms of EASI75 at week 4 but not at week 16. Significant reductions in EASI, DLQI, POEM, HADS, NRS-itch, and VAS-sleep were found between week 4 versus baseline $(p<0.0001$ for all) and week 16 versus baseline $(p<0.0001$ for all). Early AD onset and absence of hypereosinophilia may be suggested as predictive markers of early response to dupilumab. We confirmed the efficacy and safety of this agent along with the improvement of life quality in severe AD patients.
\end{abstract}

Keywords: atopic dermatitis; dupilumab; quality of life; disease severity

\section{Introduction}

Atopic dermatitis (AD) is a debilitating, chronic-relapsing inflammatory dermatosis characterized by skin xerosis, pruritic lesions, and detrimental effects on sleep, mood, productivity, and quality of life [1]. Its complex pathophysiology involves both the disruption of skin barrier function and a T helper 2 (Th2)-polarized immune response [2]. Management of mild forms of AD relies on the use of emollients, topical corticosteroids/calcineurin inhibitors, and phototherapy, while systemic immunomodulant/immunosuppressive agents such as corticosteroids and cyclosporine A (CsA) are used for severe refractory cases. Looking beyond conventional drugs, data on the newer targeted therapies, including monoclonal antibodies and oral small molecules, are very promising [3-5]. In this regard, dupilumab (Dupixent ${ }^{\circledR}$ ), a fully human monoclonal antibody inhibiting the interleukin 
(IL)-4/IL-13 signaling through the blockade of the IL-4 receptor $\alpha$ subunit, has been recently approved by both the US Food and Drug Administration (FDA) and the European Medicines Agency (EMA) for the treatment of patients with moderate-to-severe inadequately controlled AD [6]. However, although dupilumab has been demonstrated to be an effective and safe therapeutic option by the two identical phase-3 SOLO1 and SOLO2 trials [7] and the LIBERTY AD CHRONOS [8] and LIBERTY AD CAFÉ [9] phase-3 trials, literature data resulting from a real-life daily practice setting are limited [10-18].

Therefore, with the aim of identifying possible predictors of response to dupilumab and assessing the clinical response to this drug in terms of quality of life and disease severity improvement, we retrospectively assessed cases of severe AD treated with dupilumab for a period of at least 16 weeks at our Department.

\section{Materials and Methods}

\subsection{Patients}

We performed a retrospective chart review of 117 patients with severe AD treated with dupilumab for a period of at least 16 weeks from June 2018 to November 2019 at the Dermatology Department of the University of Milan, Italy. According to the Italian Drug Agency (AIFA) recommendations for dupilumab prescription, inclusion criteria were: (i) age $\geq 18$ years; (ii) severe disease defined by a baseline Eczema Area and Severity Index (EASI) $\geq 24$; (iii) inadequate response/intolerance to CsA or medical inadvisability of CsA treatment. Patients with any documented psychiatric comorbidity were excluded from the study.

All patients were treated with self-administered subcutaneous dupilumab $300 \mathrm{mg}$ every other week following a loading dose of dupilumab $600 \mathrm{mg}$ subcutaneously administered by a clinician. Traditional immunosuppressive agents (e.g., CsA, azathioprine, methotrexate) were discontinued at least 4 weeks before dupilumab initiation in all patients, while systemic corticosteroids were maintained in a minority of patients, with progressive tapering and subsequent withdrawal within 2 weeks. Concomitant topical corticosteroids or calcineurin inhibitors were allowed. Patients were evaluated three times: at baseline, 4 , and 16 weeks after dupilumab initiation.

All patients agreed with the treatment regimen and signed a written consent form to extract relevant data from their charts. In view of the retrospective nature of the study, only a notification to the Ethical Committee of the Fondazione IRCCS Ca' Granda Ospedale Maggiore Policlinico, Milan Italy) was requested.

\subsection{Demographic, Clinical, and Laboratory Features}

At baseline, the following data were collected: sex, age at AD onset, age at dupilumab initiation, extrinsic/intrinsic status, baseline total serum immunoglobulin (Ig)E, baseline serum eosinophil count, previous treatment with CsA (intolerance/ineffectiveness/contraindication), and concurrent use of systemic corticosteroids at baseline. Adult-onset AD was defined by the cut-off value of 18 years, while hypereosinophilia was defined in presence of blood eosinophil count $>500 \times 10^{3} / \mathrm{L}$. Patients were categorized in the intrinsic AD group in cases of: (i) absence of other atopic diseases such as allergic asthma and rhinoconjunctivitis, (ii) negative prick and/or intracutaneous skin tests for common inhalant and food allergens, and (iii) total serum IgE levels $\leq 200 \mathrm{kU} / \mathrm{L}$. Total serum IgE were re-assessed 4 weeks after dupilumab initiation and the ratio between week 4 and baseline total serum IgE was calculated.

\subsection{Physician- and Patient-Reported Outcomes}

Disease severity was determined at baseline and at weeks 4 and 16 after dupilumab, starting by means of the physician-reported outcome EASI [19]. Response to dupilumab was defined as an improvement of $\geq 75 \%$ in EASI from baseline (EASI75). Additionally, patient-reported outcomes including the Italian version of Dermatology Life Quality Index (DLQI) questionnaire [20], 
Patient-Oriented Eczema Measure (POEM) [21], Hospital Anxiety and Depression Scale (HADS) [22], Peak Pruritus Numerical Rating Scale (NRS-itch) during the past 7 days [23], and VAS-sleep [24], which are measurement instruments widely accepted to evaluate quality of life in AD, were collected at baseline and at weeks 4 and 16 after dupilumab initiation.

\subsection{Statistical Analysis}

Categorical variables were reported as frequencies and percentages while continuous variables were reported as medians and interquartile range (IQR). The non-parametric Wilcoxon signed-rank test was used to compare paired values of total serum IgE, physician-reported, and patient-reported outcomes between week 4 and baseline and between week 16 and baseline.

Logistic regression models were used to assess the association between response to dupilumab (EASI reduction $>75 \%$ ) and the following potential predictive factors: sex; age at onset (dichotomised as $<$ or $>18$ years); age at dupilumab initiation (dichotomised as $<$ or $>$ observed median value); previous CsA treatment; status (intrinsic versus extrinsic); hypereosinophilia ( $>500$ eosinophils $/ \mu \mathrm{L}$ ); baseline total IgE (dichotomised as $<$ or $>$ observed median value); and concurrent therapy with systemic corticosteroids. Univariate and multivariate logistic regression analyses were performed. At the first step, univariate models were fitted, considering all the variables reported above, in order to identify potentially relevant predictors to be considered in multivariate analyses. A less-restrictive $p$ value $<0.10$ was used to identify candidate predictors to be included in the multivariate analysis. Finally, a multivariate model was fitted considering only the variables identified at the univariate step. Two separate logistic regression analyses were performed considering EASI reduction at week 4 and at week 16.

$P$ values less than 0.05 , two sided, were considered statistically significant. The statistical software SAS (release 9.4, SAS Institute, Inc., Cary, NC, USA) was used to perform all the statistical analyses.

\section{Results}

\subsection{Patients' Demographic Data and Predictors of Response}

A total of 117 patients, 52 females and 65 males, met the inclusion criteria and were eligible for the study. The patients' characteristics are summarized in Table 1. They had a mean age at onset of 11 years (range: 0-76) and a median age at dupilumab initiation of 39 (IQR: 26-50). Adult-onset AD was observed in 31 (26.5\%) patients, while an intrinsic type of AD was observed in 19 (16.2\%) patients. Prior to dupilumab, CsA had been administered in 103 patients, of whom 49 (41.9\%) developed adverse events and $54(46.1 \%)$ showed an inadequate response. Conversely, 14 patients had never received CsA as it was contraindicated.

Table 1. Demographic and clinical features of the patients.

\begin{tabular}{ll}
\hline & $n=\mathbf{1 1 7}$ \\
\hline Males, $n(\%)$ & $65(55.6)$ \\
\hline Age at onset, years, mean (range) & $11(0-76)$ \\
\hline Age at dupilumab initiation, median (IQR) & $39(26-50)$ \\
\hline Early onset, $n(\%)$ & $86(73.5)$ \\
\hline Hypereosinophilia, $n(\%)$ & $50(42.7)$ \\
\hline Extrinsic AD, $n(\%)$ & $98(83.8)$ \\
\hline
\end{tabular}

Median baseline eosinophil count was 350/ $\mu \mathrm{L}(\mathrm{IQR}: 120-690 / \mu \mathrm{L})$ and $50(42.7 \%)$ patients were classified as having hypereosinophilia. Median baseline total IgE were $1809 \mathrm{kU} / \mathrm{L}$ (IQR: 338-4210 kU/L; mean: $4210.2 \mathrm{kU} / \mathrm{L}$ ) and median week 4 total IgE were 1938 (IQR:242-4680 kU/L; mean $3602.3 \mathrm{kU} / \mathrm{L}$ ) [see Table 1]. Median ratio between baseline and week 4 total IgE was 1.27 (IQR:1.08-1.65; mean: 
1.89). Twenty-three (19.7\%) patients were maintained on systemic corticosteroids during dupilumab treatment, with progressive tapering and steroid discontinuation within 3 weeks in all cases. The statistical analysis showed no association between w4IgE/bIgE ratio and the EASI variation between baseline and week $4(p=0.21)$.

As shown in Table 2, sex, intrinsic versus extrinsic status, baseline serum total IgE (dichotomised using the median value, that is $<$ or $>=1809 \mathrm{kU} / \mathrm{L}$ ), early dupilumab initiation, and intolerance/contraindication versus ineffectiveness of CsA were not important predictors of response to dupilumab in terms of EASI75 either at week 4 and at week 16. Interestingly, at multivariate analysis early $\mathrm{AD}$ onset [OR, 2.9; 95\% CI, 1.2-7.2; $p=0.0207]$ and absence of hypereosinophilia [OR, $2.24 ; 95 \%$ CI 1.03-4.86; $p=0.0412)]$ were identified as significant predictive parameters for response to dupilumab in terms of EASI75 at week 4 . At week 16, no predictive parameters were significantly associated with response to dupilumab. Considering the multivariate model including the two factors with $p<0.1$ at univariate analysis at week 4 , we found that early AD onset confirmed the statistical significance at multivariate analysis. 
Table 2. Odds ratios (OR) and 95\% confidence intervals (CI) for response to dupilumab in terms of EASI75 according to baseline clinical parameters in patients with severe atopic dermatitis at week 4 and week 16.

\begin{tabular}{|c|c|c|c|c|c|c|c|}
\hline & & \multicolumn{3}{|c|}{ Week $4 \S$} & \multicolumn{3}{|c|}{ Week 16} \\
\hline & & OR & $95 \% \mathrm{CI}$ & $p$ Value & OR & $95 \%$ CI & $p$ Value \\
\hline \multirow{2}{*}{ Sex } & Females & $1^{*}$ & & \multirow{2}{*}{1} & $1^{*}$ & & \multirow{2}{*}{0.355} \\
\hline & Males & 1 & $0.5-2.1$ & & 0.7 & $0.3-1.6$ & \\
\hline \multirow{2}{*}{ Early/adult-onset (< or >18 years) } & Adult-onset & $1 *$ & & \multirow{2}{*}{0.029} & $1 *$ & & \multirow{2}{*}{0.239} \\
\hline & Early-onset & 2.7 & $1.1-6.5$ & & 1.7 & $0.7-4.1$ & \\
\hline \multirow{2}{*}{ Age at dupilumab initiation } & $>39$ & $1^{*}$ & & \multirow{2}{*}{0.493} & $1^{*}$ & & \multirow{2}{*}{0.585} \\
\hline & $<=39$ & 1.3 & $0.6-2.7$ & & 0.8 & $0.4-1.8$ & \\
\hline \multirow{2}{*}{ Previous cyclosporine A treatment } & Contraindication or intolerance & $1 *$ & & \multirow{2}{*}{0.728} & $1 *$ & & \multirow{2}{*}{0.251} \\
\hline & Ineffectiveness & 1.2 & $0.6-2.4$ & & 1.6 & $0.7-3.7$ & \\
\hline \multirow{2}{*}{ Intrinsic versus extrinsic status } & Intrinsic & $1^{*}$ & & \multirow{2}{*}{0.7} & $1^{*}$ & & \multirow{2}{*}{0.652} \\
\hline & Extrinsic & 1.2 & $0.5-3.3$ & & 1.3 & $0.4-3.7$ & \\
\hline \multirow{2}{*}{ Hypereosinophilia (>500 eosinophils/ $\mu \mathrm{L}$ ) } & Yes & $1^{*}$ & & \multirow{2}{*}{0.059} & $1^{*}$ & & \multirow{2}{*}{0.579} \\
\hline & No & 2.1 & $1-4.4$ & & 1.3 & $0.6-2.9$ & \\
\hline \multirow{2}{*}{ Baseline total $\operatorname{IgE}(>1809 \mathrm{KU} / \mathrm{L})$} & No & $1 *$ & & \multirow{2}{*}{0.776} & $1^{*}$ & & \multirow{2}{*}{0.440} \\
\hline & Yes & 0.9 & $0.4-1.9$ & & 1.4 & $0.6-3.1$ & \\
\hline \multirow{2}{*}{ Concurrent therapy with systemic corticosteroids } & No & $1 *$ & & \multirow{2}{*}{0.347} & $1^{*}$ & & \multirow{2}{*}{0.891} \\
\hline & Yes & 1.5 & $0.7-3.3$ & & 1.3 & $0.4-2.6$ & \\
\hline
\end{tabular}

* Reference category. § At multivariate analysis, including "early/adult-onset" and "hypereosinophilia", the following results were obtained: "early/adult-onset": OR for early onset: 2.91 (95\% CI 1.18-7.18; $p=0.0207)$, “hypereosinophilia": OR for absence of hypereosinophilia: 2.24 (95\% CI 1.03-4.86; $p=0.0412$ ). 


\subsection{Disease Severity}

Median baseline EASI was 30 (IQR: 24-37) and significantly dropped to 8 (IQR: 5-12; $p<0.001$ ) at week 4. A further reduction of median EASI was observed at week 16, when its value was 5 (IQR: $2-8)$. This reduction was statistically significant with respect to both week $4(p<0.001)$ and baseline $(p<0.001)$ [see Table 1]. Fifty-four out of $117(46.2 \%)$ patients achieved EASI75 at week 4, while at week 16, patients who achieved EASI75 raised to 85/117 (72.7\%).

\subsection{Quality of Life}

As shown in Table 3, the median DLQI score, which was 16 at baseline, dropped to 5 at week 4 and to 3 at week 16. The median POEM score, which was 23 at baseline, dropped to 9 at week 4 and to 6 at week 16. We found a statistically significant reduction in DLQI scores between week 4 versus baseline $(p<0.0001)$ and week 16 versus baseline $(p<0.0001)$. Likewise, we found a statistically significant reduction in POEM scores of week 4 versus baseline $(p<0.0001)$ and week 16 versus baseline $(p<0.0001)$. Median HADS-anxiety at baseline was 8 (IQR:5-11) and dropped to 4 (IQR: 2-6) at week 4 and to 3 (IQR: 1-5) at week 16. Similarly, HADS-depression dropped from 7 (IQR: 4-10) at baseline to 4 (IQR: 1-7) at week 4 and to 3 (IQR: 0-6) at week 16. Median NRS-itch, which was 9 (IQR: 8-10) at baseline, dropped to 4 (IQR: 2-5) at week 4 and to 3 (IQR: $1-4$ ) at week 16, while median VAS-sleep was 8 (IQR: 5-10) at baseline and dropped to 1 (IQR: 0-3) at week 4 and to 0 (IQR: 0-1) at week 16. Statistically significant differences were observed either for HADS-anxiety, HADS-depression, NRS-itch, and VAS-sleep when comparing values of week 4 versus baseline $(p<0.0001)$ and week 16 versus baseline $(p<0.0001)$.

Table 3. Total serum IgE, physician-reported, and patient-reported outcomes at baseline, week 4, and week 16 after dupilumab starting.

\begin{tabular}{llllll}
\hline & Baseline & Week 4 & $p$ Value & Week 16 & $p$ Value $^{* *}$ \\
\hline Serum total IgE & $1809(338-5434)$ & $1938(242-4680)$ & $<0.0001$ & - & - \\
\hline EASI & $30(24-37)$ & $8(5-12)$ & $<0.0001$ & $5(2-8)$ & $<0.0001$ \\
\hline POEM & $23(18-27)$ & $9(5-12)$ & $<0.0001$ & $6(3-10)$ & $<0.0001$ \\
\hline DLQI & $16(12-22)$ & $5(3-10)$ & $<0.0001$ & $3(1-7)$ & $<0.0001$ \\
\hline HADS-depression & $7(5-10)$ & $4(2-7)$ & $<0.0001$ & $3(1-5.5)$ & $<0.0001$ \\
\hline HADS-anxiety & $8(5-11)$ & $4(2-6)$ & $<0.0001$ & $3(1-5)$ & $<0.0001$ \\
\hline NRS-itch & $9(8-10)$ & $4(2-5)$ & $<0.0001$ & $3(1-4)$ & $<0.0001$ \\
\hline VAS sleep & $8(5-10)$ & $1(0-3)$ & $<0.0001$ & $0(0-1)$ & $<0.0001$ \\
\hline
\end{tabular}

Data are reported as median (interquartile range). ${ }^{*}$ comparison between week 4 and baseline. ${ }^{* *}$ comparison between week 16 and baseline.

\subsection{Safety}

The majority of adverse events were mild in severity and included blepharoconjunctivitis $(n=14$; $11.9 \%)$, facial redness $(n=6 ; 5.1 \%)$, and paradoxical psoriasis $(n=1 ; 0.8 \%)$. The severity of the adverse event led to drug discontinuation only in a patient with blepharoconjunctivitis and in the patient with paradoxical psoriasis at week 24 and week 32, respectively.

\section{Discussion}

This retrospective single-center study on a cohort of 117 patients with severe AD reflects the Italian real-life experience in the management of the disease with dupilumab and confirms the efficacy of this agent for refractory cases. Indeed, considering that our study population is similar to that of the LIBERTY AD CAFE study, being represented by patients with severe AD refractory to CsA, 
approximately $70 \%$ of our patients, in line with most studies present in the literature [8-10], achieved EASI75 at week 16 after starting dupilumab.

A statistically significant reduction in EASI score was achieved from baseline at week 4 and week 16 after starting dupilumab. As of now, some of the patients included in the study achieved week 52, maintaining a good response and low EASI score (unpublished data). The clinical improvement resulting from the physician-reported outcome EASI was accompanied by a considerable amelioration of quality of life, witnessed by a significant reduction of patient-reported outcomes widely used in AD such as DLQI, POEM, NRS-itch, and VAS-sleep from baseline both at week 4 and week 16 [25-27]. In addition, we investigated anxiety and depression using HADS and we substantiated, in accordance with a previous study by Cork et al. [28], a significant reduction in anxiety and depression scores after dupilumab administration.

The most remarkable finding in our study is that patients with an early AD onset seemed to respond better to dupilumab at week 4 . The confirmation of this result in the multivariate analysis, where we decided to include variables with $p$ value $<0.1$ in the univariate analysis (instead of the usual value of $p<0.05$ ), emphasizes the importance of early AD onset as a predictor of response to dupilumab. Even if at week 16 we failed to confirm this association, early onset showed a certain association $(\mathrm{OR}=1.7)$ with response in terms of EASI75, albeit the result was not statistically significant. This may be due to the relatively small sample size, with consequent low statistical power.

Furthermore, the result indicating absence of hypereosinophilia as a predictive biomarker of response $(\mathrm{OR}=2.24 ; p=0.0412)$ in the multivariate analysis at week 4 suggests a possible role of eosinophils in response to dupilumab. Indeed, inhibition of group 2 innate lymphoid (ILC2) cells and, consequently, eosinophils may be one of the mechanisms of action of dupilumab [29]. However, further experimental and clinical studies are needed to confirm this hypothesis.

In our cohort, extrinsic status and baseline total IgE was not correlated with response to dupilumab both at week 4 and at week 16. Moreover, as expected [30], although a slight decrease of total IgE was observed in the first 4 weeks of treatment, the ratio between week 4 and baseline total IgE did not correlate with dupilumab response in terms of EASI75.

Finally, we also showed for the first time that patients who experienced ineffectiveness of a previous course of CsA had similar response to dupilumab as compared to patients with contraindication or intolerance to this drug, suggesting that response to CsA does not influence the effectiveness of dupilumab.

Dupilumab was well tolerated in most patients, with only $11.9 \%$ of patients suffering from blepharoconjunctivitis, a finding which is in contrast to studies reporting eye symptoms in up to $62 \%$ of cases [18]. Although the underlying mechanism of blepharoconjunctivitis in AD patients is still not completely elucidated, some authors have suggested that the blockade of IL-4 and IL-13 may increase the activity of specific ligands, such as OX40 ligand, involved in atopic keratoconjunctivitis [31]. It can be assumed that the relatively low incidence of this side effect might be due to the systematic use of lipid emulsion eye drops combined with hyaluronic acid eye drops in all patients from the first day of dupilumab. All patients with blepharoconjunctivitis were seen by an ophthalmologist when symptoms occurred. Blepharoconjuctivitis was the most common adverse event associated with dupilumab and according to the literature [11,32], it was successfully managed with chloramphenicol/bethametasone $0.2+0.5 \%$ eye drops. In resistant cases, we introduced tacrolimus $0.03 \%$ eye ointment. Blepharoconjuctivitis led to drug withdrawal only in cases resistant to topical treatment. Injection-site reactions were not reported by our patients. Limitations of the study are its retrospective nature, the small sample size, and the short follow-up. We are aware of the fact that these data may differ from other AD referral centers.

To sum up, our real-life study confirmed that dupilumab is an effective treatment in most patients with severe $\mathrm{AD}$ and identified early $\mathrm{AD}$ onset and absence of hypereosinophilia as predictive markers of early response to this agent. 
Author Contributions: Conceptualization, S.F. and G.G.; Data curation, G.C. and S.T.; Formal analysis, G.C., L.A., S.T., and F.G.; Investigation, F.G.; Methodology, S.F. and G.G.; Project administration, S.F.; Resources, L.A.; Software, G.C.; Supervision, E.B. and A.V.M.; Validation, G.G.; Visualization, G.G.; Writing-original draft, G.G.; Writing-review \& editing, E.B. and A.V.M. All authors have read and agree to the published version of the manuscript.

Funding: This research received no external funding.

Conflicts of Interest: The authors declare no conflict of interest.

\section{References}

1. Fishbein, A.B.; Silverberg, J.I.; Wilson, E.J.; Ong, P.Y. Update on Atopic Dermatitis: Diagnosis, Severity Assessment, and Treatment Selection. J. Allergy Clin. Immunol. Pract. 2019. [CrossRef] [PubMed]

2. Tsakok, T.; Woolf, R.; Smith, C.H.; Weidinger, S.; Flohr, C. Atopic dermatitis: the skin barrier and beyond. Br. J. Dermatol. 2019, 180, 464-474. [CrossRef] [PubMed]

3. Wollenberg, A.; Barbarot, S.; Bieber, T.; Christen-Zaech, S.; Deleuran, M.; Fink-Wagner, A.; Gieler, U.; Girolomoni, G.; Lau, S.; Muraro, A.; et al. Consensus-based European guidelines for treatment of atopic eczema (atopic dermatitis) in adults and children: part II. J. Eur. Acad. Dermatol. Venereol. 2018, 32, 850-878. [CrossRef] [PubMed]

4. Wollenberg, A.; Barbarot, S.; Bieber, T.; Christen-Zaech, S.; Deleuran, M.; Fink-Wagner, A.; Gieler, U.; Girolomoni, G.; Lau, S.; Muraro, A.; et al. Consensus-based European guidelines for treatment of atopic eczema (atopic dermatitis) in adults and children: part I. J. Eur. Acad. Dermatol. Venereol. 2018, 32, 657-682. [CrossRef] [PubMed]

5. Fabbrocini, G.; Napolitano, M.; Megna, M.; Balato, N.; Patruno, C. Treatment of Atopic Dermatitis with Biologic Drugs. Dermatol. Ther. (Heidelb.) 2018, 8, 527-538. [CrossRef] [PubMed]

6. Gooderham, M.J.; Hong, H.C.; Eshtiaghi, P.; Papp, K.A. Dupilumab: A review of its use in the treatment of atopic dermatitis. J. Am. Acad. Dermatol. 2018, 78, S28-S36. [CrossRef]

7. Simpson, E.L.; Bieber, T.; Guttman-Yassky, E.; Beck, L.A.; Blauvelt, A.; Cork, M.J.; Silverberg, J.I.; Deleuran, M.; Kataoka, Y.; Lacour, J.P. Two Phase 3 Trials of Dupilumab versus Placebo in Atopic Dermatitis. N. Engl. J. Med. 2016, 375, 2335-2348. [CrossRef]

8. Blauvelt, A.; de Bruin-Weller, M.; Gooderham, M.; Cather, J.C.; Weisman, J.; Pariser, D.; Simpson, E.L.; Papp, K.A.; Hong, H.C.; Rubel, D.; et al. Long-term management of moderate-to-severe atopic dermatitis with dupilumab and concomitant topical corticosteroids (LIBERTY AD CHRONOS): A 1-year, randomised, double-blinded, placebo-controlled, phase 3 trial. Lancet 2017, 389, 2287-2303. [CrossRef]

9. De Bruin-Weller, M.; Thaçi, D.; Smith, C.H.; Reich, K.; Cork, M.J.; Radin, A.; Zhang, Q.; Akinlade, B.; Gadkari, A.; Eckert, L.; et al. Dupilumab with concomitant topical corticosteroid treatment in adults with atopic dermatitis with an inadequate response or intolerance to ciclosporin A or when this treatment is medically inadvisable: a placebo-controlled, randomized phase III clinical trial (LIBERTY AD CAFÉ). Br. J. Dermatol. 2018, 178, 1083-1101.

10. Olesen, C.M.; Holm, J.G.; Nørreslet, L.B.; Serup, J.V.; Thomsen, S.F.; Agner, T. Treatment of atopic dermatitis with dupilumab: experience from a tertiary referral centre. J. Eur. Acad. Dermatol. Venereol. 2019, 33, 1562-1568. [CrossRef]

11. Ariëns, L.F.; van der Schaft, J.; Bakker, D.S.; Balak, D.; Romeijn, M.L.E.; Kouwenhoven, T.; Kamsteeg, M.; Giovannone, B.; Drylewicz, J.; van Amerongen, C.C.A.; et al. Dupilumab is very effective in a large cohort of difficult-to-treat adult atopic dermatitis patients: First clinical and biomarker results from the BioDay registry. Allergy 2019, 75, 116-126.

12. Faiz, S.; Giovannelli, J.; Podevin, C.; Jachiet, M.; Bouaziz, J.D.; Reguiai, Z.; Nosbaum, A.; Lasek, A.; Ferrier le Bouedec, M.C.; Du Thanh, A.; et al. Effectiveness and safety of dupilumab for the treatment of atopic dermatitis in a real-life French multicenter adult cohort. J. Am. Acad. Dermatol. 2019, 81, 143-151. [CrossRef]

13. Tauber, M.; Apoil, P.A.; Richet, C.; Laurent, J.; De Bonnecaze, G.; Mouchon, E.; Cassagne, M.; Marguery, M.C.; Hegazy, S.; Konstantinou, M.P.; et al. Effect of dupilumab on atopic manifestations in patients treated for atopic dermatitis in real-life practice. Br. J. Dermatol. 2019, 180, 1551-1552. [CrossRef] 
14. Pistone, G.; Tilotta, G.; Gurreri, R.; Castelli, E.; Curiale, S.; Bongiorno, M.R. Real-life practice: rapid improvement in itch symptomatology in patients with atopic dermatitis treated with dupilumab. J. Dermatolog. Treat. 2019. [CrossRef]

15. Ruiz-Villaverde, R.; Dominguez-Cruz, J.; Armario-Hita, J.C.; Martinez-Pilar, L.; Alcantara-Luna, S.; Pereyra-Rodriguez, J.J. Fifty-two week follow-up safety and effectiveness results of dupilumab treatment of moderate-to-severe atopic dermatitis from a retrospective, multicentric series. Dermatol. Ther. 2019, 32, e12931. [CrossRef]

16. Wang, C.; Kraus, C.N.; Patel, K.G.; Ganesan, A.K.; Grando, S.A. Real-world experience of dupilumab treatment for atopic dermatitis in adults: a retrospective analysis of patients' records. Int. J. Dermatol. 2020, 59, 253-256. [CrossRef]

17. Armario-Hita, J.C.; Pereyra-Rodriguez, J.; Silvestre, J.F.; Ruiz-Villaverde, R.; Valero, A.; Izu-Belloso, R.; Jáuregui-Presa, I.; Curto-Barredo, L.; Figueras-Nart, I.; Herranz-Pinto, P.; et al. Treatment of moderate-to-severe atopic dermatitis with dupilumab in real clinical practice: a multicentre, retrospective case series. Br. J. Dermatol. 2019, 181, 1072-1074. [CrossRef]

18. De Wijs, L.E.M.; Bosma, A.L.; Erler, N.S.; Hollestein, L.M.; Gerbens, L.A.A.; Middelkamp-Hup, M.A.; Kunkeler, A.C.M.; Nijsten, T.E.C.; Spuls, P.I.; Hijnen, D.J. Effectiveness of dupilumab treatment in 95 patients with atopic dermatitis: daily practice data. Br. J. Dermatol. 2020, 182, 418-426. [CrossRef] [PubMed]

19. Hanifin, J.M.; Thurston, M.; Omoto, M.; Cherill, R.; Tofte, S.J.; Graeber, M. The eczema area and severity index (EASI): assessment of reliability in atopic dermatitis. EASI Eval. Group. Exp. Dermatol. 2001, 10, 11-18. [CrossRef] [PubMed]

20. Finlay, A.Y.; Khan, G.K. Dermatology Life Quality Index (DLQI)—a simple practical measure for routine clinical use. Clin. Exp. Dermatol. 1994, 19, 210-216. [CrossRef] [PubMed]

21. Charman, C.R.; Venn, A.J.; Williams, H.C. The patient-oriented eczema measure: development and initial validation of a new tool for measuring atopic eczema severity from the patients' perspective. Arch. Dermatol. 2004, 140, 1513-1519. [CrossRef] [PubMed]

22. Silverberg, J.I.; Gelfand, J.M.; Margolis, D.J.; Boguniewicz, M.; Fonacier, L.; Grayson, M.H.; Ong, P.Y.; Chiesa Fuxench, Z.C.; Simpson, E.L. Symptoms and diagnosis of anxiety and depression in atopic dermatitis in U.S. adults. Br. J. Dermatol. 2019, 181, 554-565. [CrossRef] [PubMed]

23. Yosipovitch, G.; Reaney, M.; Mastey, V.; Eckert, L.; Abbé, A.; Nelson, L.; Clark, M.; Williams, N.; Chen, Z.; Ardeleanu, M.; et al. Peak Pruritus Numerical Rating Scale: psychometric validation and responder definition for assessing itch in moderate-to-severe atopic dermatitis. Br. J. Dermatol. 2019, 181, 761-769. [CrossRef] [PubMed]

24. Tsianakas, A.; Luger, T.A.; Radin, A. Dupilumab treatment improves quality of life in adult patients with moderate-to-severe atopic dermatitis: results from a randomized, placebo-controlled clinical trial. Br. J. Dermatol. 2018, 178, 406-414. [CrossRef]

25. Barrett, A.; Hahn-Pedersen, J.; Kragh, N.; Evans, E.; Gnanasakthy, A. Patient-Reported Outcome Measures in Atopic Dermatitis and Chronic Hand Eczema in Adults. Patient 2019, 12, 445-459. [CrossRef]

26. Silverberg, J.I.; Margolis, D.J.; Boguniewicz, M.; Fonacier, L.; Grayson, M.H.; Ong, P.Y.; Fuxench, Z.C.; Simpson, E.L. Validation of five patient-reported outcomes for atopic dermatitis severity in adults. Br. J. Dermatol. 2020, 182, 104-111. [CrossRef] [PubMed]

27. Chalmers, J.R.; Simpson, E.; Apfelbacher, C.J.; Williams, H.C.; Prinsen, C.A.; Spuls, P.I.; Simpson, E.; Gerbens, L.A.A.; Boers, M.; Barbarot, S.; et al. Report from the 4th international consensus meeting to harmonize core outcome measures for atopic eczema/dermatitis clinical trials. Br. J. Dermatol. 2016, 175, 69-79. [CrossRef]

28. Cork, M.J.; Eckert, L.; Simpson, E.L.; Armstrong, A.; Barbarot, S.; Puig, L.; Girolomoni, G.; de Bruin-Weller, M.; Wollenberg, A.; Kataoka, Y.; et al. Dupilumab improves patient-reported symptoms of atopic dermatitis, symptoms of anxiety and depression, and health-related quality of life in moderate-to-severe atopic dermatitis: analysis of pooled data from the randomized trials SOLO 1 and SOLO 2. J. Dermatolog. Treat. 2019. [CrossRef]

29. Patel, G.; Pasha, M.A.; D'Souza, S. Group 2 Innate Lymphoid Cells in Patients with Severe Atopic Dermatitis on Dupilumab. J. Allergy Clin. Immunol. 2019, 143, 2. [CrossRef] 
30. Guttman-Yassky, E.; Bissonnette, R.; Ungar, B.; Suárez-Fariñas, M.; Ardeleanu, M.; Esaki, H.; Suprun, M.; Estrada, Y.; Xu, H.; Peng, X.; et al. Dupilumab progressively improves systemic and cutaneous abnormalities in patients with AD. J. Allergy Clin. Immunol. 2019, 143, 155-172. [CrossRef]

31. Mennini, M.; Dahdah, L.; Fiocchi, A. Two Phase 3 Trials of Dupilumab versus Placebo in Atopic Dermatitis. N. Engl. J. Med. 2017, 376, 1090. [CrossRef] [PubMed]

32. Wollenberg, A.; Ariens, L.; Thurau, S.; van Luijk, C.; Seegräber, M.; de Bruin-Weller, M. Conjunctivitis occurring in atopic dermatitis patients treated with dupilumab-clinical characteristics and treatment. J. Allergy Clin. Immunol. Pract. 2018, 6, 1778-1780. [CrossRef] [PubMed]

(C) 2020 by the authors. Licensee MDPI, Basel, Switzerland. This article is an open access article distributed under the terms and conditions of the Creative Commons Attribution (CC BY) license (http://creativecommons.org/licenses/by/4.0/). 